\title{
Impedance of Adhesive Joints
}

\author{
Pavel Mach, Jiří Koláŕ \\ Department of Electrotechnology, Faculty of Electrical Engineering, \\ Czech Technical University in Prague \\ Technická 2, 16627 Prague 6, Czech Republic \\ mach@fel.cvut.cz
}

\begin{abstract}
Impedance of adhesive joints has been measured in frequency range $20 \mathrm{~Hz}-1 \mathrm{MHz}$ using LCR meter HP 4284A. The joints have been created by adhesive assembly of jumpers on test PCBs. It has been found that the joint impedance increases at the frequency higher than $100 \mathrm{kHz}$. It has been found that the measured frequency characteristics are in correlation with frequency characteristic of an equivalent circuit diagram consisting of in-series connected resistor and inductor.
\end{abstract}

\section{INTRODUCTION}

Impedance is an important parameter used to characterize electrical circuits, components, and materials for components fabrication. Impedance is generally defined as the total opposition a device offers to the flow of an alternating current at a given frequency, and is represented as a complex quantity which is graphically shown on a vector plane.

Impedance vector consists of a real part (resistance $\mathrm{R}$ ) and an imaginary part (reactance $\mathrm{X}$ ). Impedance can be expressed as sum of the real and imaginary part.

The reciprocal of impedance is admittance. Admittance can be calculated as sum of conductance and susceptance, where conductance is a real part of admittance and susceptance is an imaginary part.

Adhesive joining is used in some applications to avoid to high temperature, which must be used during a soldering process. Electrical conductivity of adhesives is caused by a conductive net created by particles of filler, which are added into resin, used as a binder. Resin is an insulating material. Mostly bis phenol epoxy is used as a binder and silver flakes as a filler.

Electrical conductivity of adhesives is dominantly influenced by two mechanisms: by the constriction resistance and by tunneling. The constriction resistance is caused by a small contact area between particles in comparison with their dimensions. Tunneling is caused by thin insulating films, which cover surfaces of filler particles.

The total resistance of conductive joints formed of electrically conductive adhesives is meaningfully influenced by the constriction resistance of contacts between particles, whereas the total resistance of joints formed of adhesives filled with flakes is slightly influenced only by this constriction resistance and tunneling resistance dominates.

The resistance of adhesive joints depends on density of conductive net created inside adhesive after curing and on quality of contacts between conductive particles.

Frequency dependence of impedance of adhesive joints is a very significant parameter, which can limit the use of adhesives in high frequency applications. The goal of the work is to examine impedance of adhesive joints in a wide frequency spectrum.

\section{IMPEDANCE OF ELECTRONIC COMPONENTS}

To measure the impedance it is necessary to measure two quantities: the real part and the imaginary part. Contemporary impedance measuring equipment measures these two parts and converts them into requested quantity, mostly into absolute value of impedance or admittance. Measurement accuracy for variety of impedance parameters depends on chosen measurement method. 
Resistors, capacitors and inductors are usually represented by their nominal values, it means by the resistance, capacitance or inductance [1].

It is necessary to accept that all real-word passive components have parasitics, e.g. unwanted inductance in resistors, capacitance in inductors, inductances in capacitors etc. Different materials produce different level of parasitics. Combination of a component's primary element and parasitics, a component is like a complex circuit. It means that every component must have higher or lower frequency dependency of impedance. Not all parasitics affect the measurement, but some of them determine the shape of the frequency characteristic of a component.

Frequency dependency is common to all real-word components because of the existence of parasitics.

Adhesive joints are components with dominant resistive character and with low resistance. With respect to conductive net inside adhesive it is possible to assume that an equivalent circuit diagram will consist of in-series connected resistor and inductor. The resistance is dominantly given by the tunneling resistance of contacts between filler particles (if silver flakes are used as a filler); the conductive net represents "leads" to these contact resistors. The frequency dependence of such the component is shown in Fig. 1 [1].

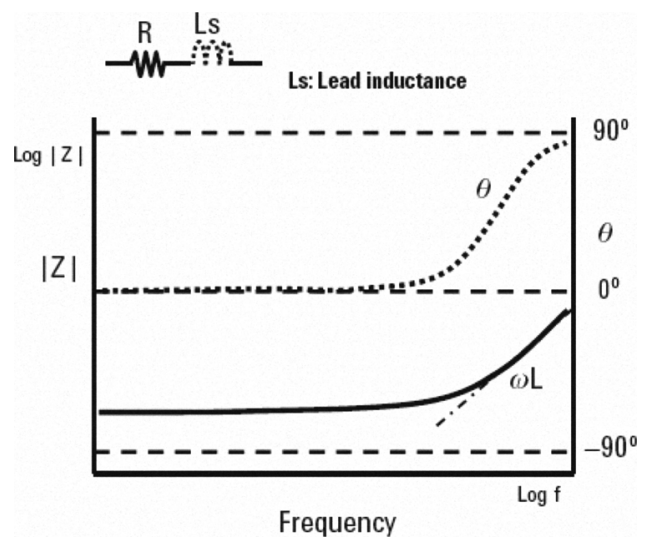

Fig. 1 Frequency dependence of phase angle and impedance of low-value resistors

It follows from Fig. 1 that starting from some frequency the impedance of a low-value resistor starts to grow. The reason is that some parasitic capacitance is also present and the impedance starts to grow to the self- resonant frequency of the resistor.

\section{MeAsuring of AdHesive JoInts IMPEDANCE}

The impedance measurements are carried out using an AC signal. The amplitude of the signal can influence results of measurement. On the one hand, this amplitude cannot be too high, because high amplitude can cause heating of measured components and distort results of measurement. On the other hand, if the amplitude of the measuring signal would be too low, sensitivity of measuring equipment will limit the measurement quality and results could also be distort by noise.

Qualification of impedances and recommended equivalent circuit diagram are shown in Fig. 2 [1].

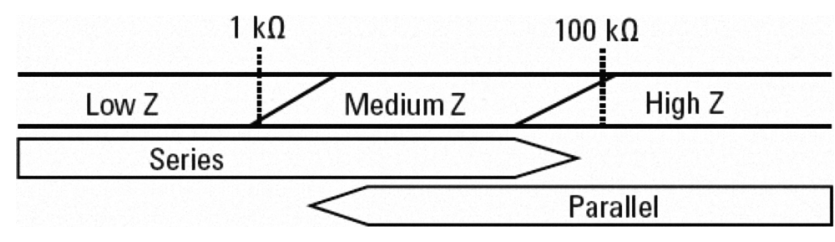

Fig. 2 High and low impedance criteria

Most types of components, including adhesive joints, are temperature dependent. The temperature coefficient is a very significant specification for resistors (including low-value resistors such like adhesive joints), capacitors and inductors.

Other physical, electrical and chemical environments, e.g. humidity, mechanical stress, salt fog and others may also influence the impedance. Especially, as for electrically conductive adhesives, humidity is environment, which can change electrical and mechanical properties of adhesive joints meaningfully.

In frequency range, where the adhesive joint exhibits almost a flat impedance characteristic, either a series or parallel equivalent circuit can be applied as a suitable model to express impedance characteristic. The simple series or parallel models are effective for such the description.

The measurements have been carried out using a precision LCR meter Agilent 4284A. To minimize influence of leads between measured joint and measuring equipment a special fixture has been designed and realized. The test PCBs with adhesive assembly mounted jumpers have been contacted in the measuring fixture and located inside a screening box 
for the impedance measurement. Jumpers have been fabricated as silver cubes with dimensions $1,5 \times 1,5 \times 1,5 \mathrm{~mm}$. Output connectors of the fixture have been directly joined with input connectors of the LCR meter, without connecting cables to minimize inductance of connections. The measurement has been carried out in four-terminal configuration. The schematic diagram of this arrangement is in Fig. 3.

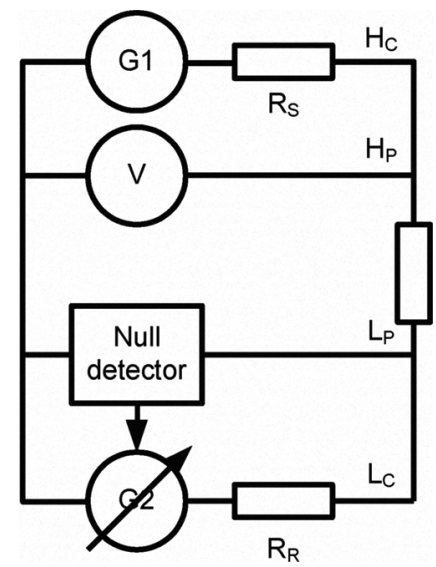

Fig. 3 Four-terminal configuration of impedance measurement

Connection of connectors of the LCR meter is shown in Fig. 4.

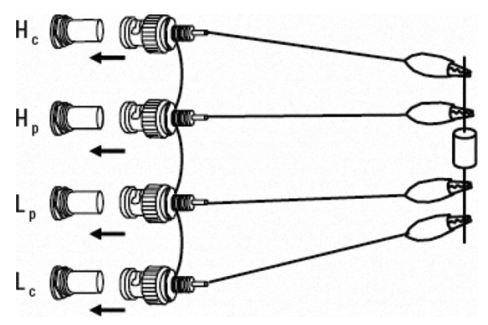

Fig. 4 Connection of connectors of the measuring equipment with measured joint (joint is schematically represented with a component)

The powering of the measured joint has been carried out by the voltage of $0,5 \mathrm{mV}$. The current flowing through the joint is approximately $25 \mathrm{~mA}$. It is found that such the current intensity does not cause significant heating of the joint. Heating has been tested during long-time measurement of the impedance. It has been found that impedance does not change during the time longer than 60 seconds if the measuring signal is connected. Arrangement of measuring fixture is shown in Fig. 5.

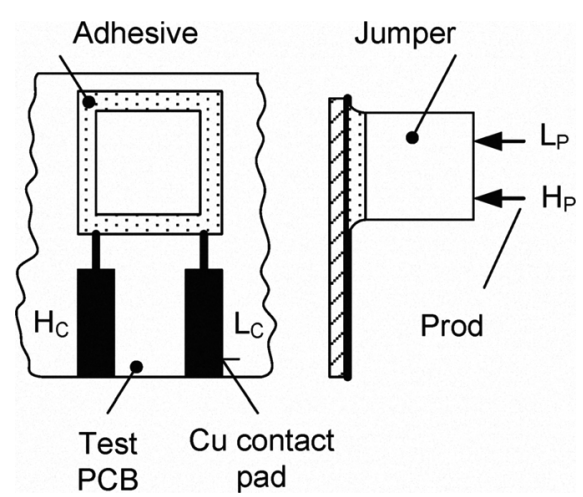

Fig. 5 Fixture for measurement of adhesive joints impedance

Adhesive joints have been formed of 8 types of electrically conductive adhesives. All formulations have been based on bis phenol epoxy filled with silver flakes. Curing temperatures have been from $120^{\circ} \mathrm{C}$ to $140{ }^{\circ} \mathrm{C}$, curing times from 20 to $60 \mathrm{~min}$.

\section{Results of Measurements}

The measurements have been carried out in the frequency range $20 \mathrm{~Hz}-1 \mathrm{MHz}$. Real part, imaginary part, and absolute value of impedance has been examined. The results of the measurements are shown in Fig. 6 to Fig. 9

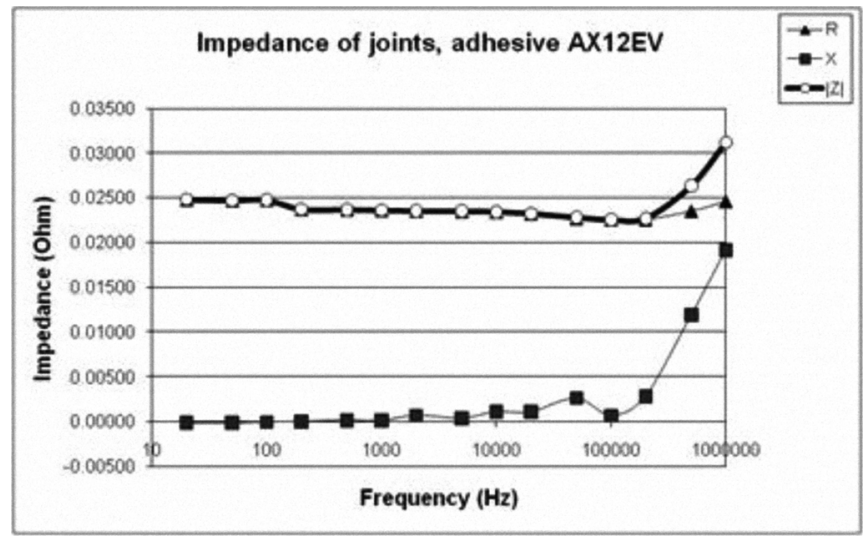

Fig. 6 Impedance of the joint formed of adhesive AX12E 


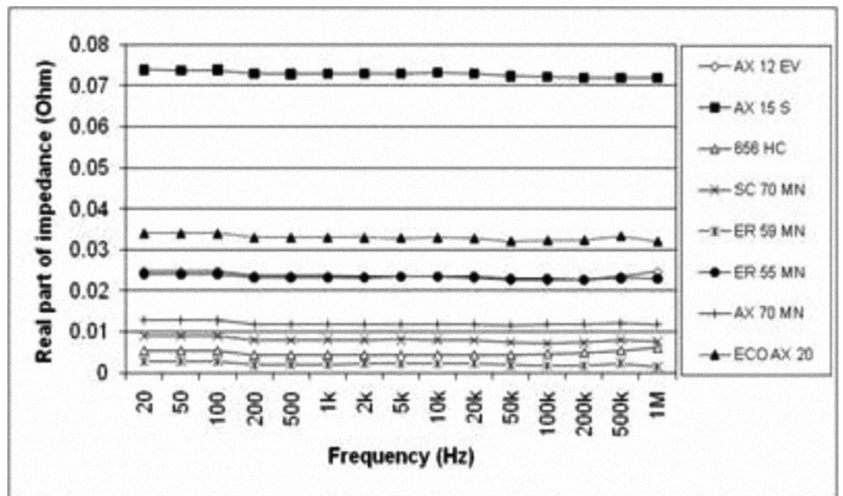

Fig. 7 Real part of impedance of adhesive joints formed of different types of adhesives

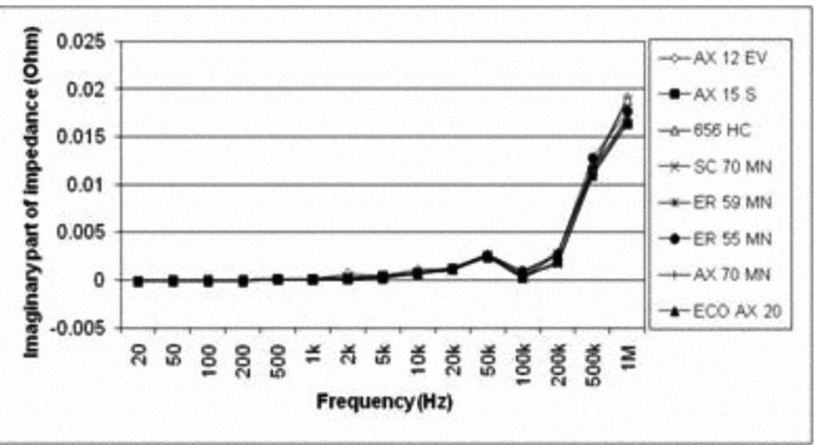

Fig. 8 Imaginary part of impedance of adhesive joints formed of different types of adhesives

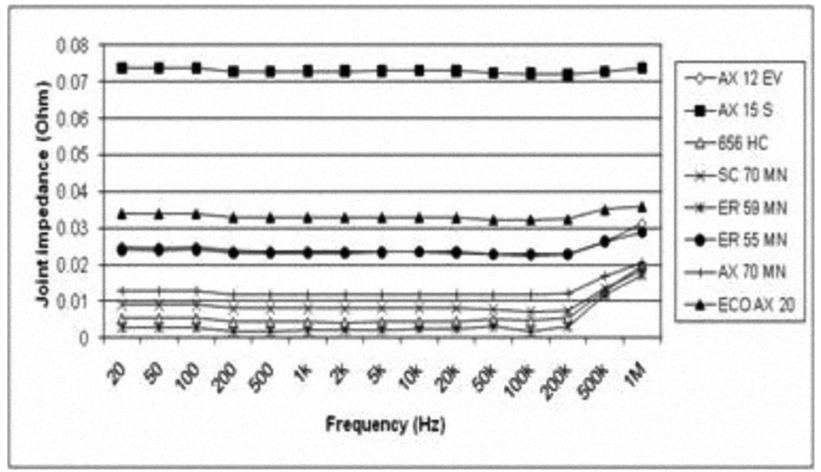

Fig. 9 Impedance of adhesive joints formed of different types of adhesives

\section{Discussion AND CONCLUSIONS}

It has been found that the equivalent circuit, which can be used for simulation of frequency characteristic of adhesive joints, is simple series connection of the resistor and inductor. The value of the inductor can be calculated from the slope of the frequency characteristic. The break of the frequency characteristic starts over the frequency of $100 \mathrm{kHz}$. To this frequency the impedance can be taken constant.

The reason of this character of the adhesive joints impedance is a conductive net created inside adhesive binder after curing. Curing causes partial sintering of conductive particles and creates the net.

With respect to the basic structure of conductive net inside adhesive it has been assumed, that the equivalent circuit for simulation of frequency dependence of the joint will be in-series connected resistor and impedance. This assumption has been conferment by the measurement.

It has also been found that the measurement of the frequency dependence of impedance of adhesive joints is possible with sufficient accuracy. However, the measuring workplace must be sufficiently screened and fixture, which minimizes length of connection of measured joints with input connectors of the measuring equipment, has to be used.

Frequency dependence of adhesive joints formed of 8 types of electrically conductive adhesives has been measured. The dependences are very similar due to similar formulations used for binder and similar types and materials of filler particles.

The results are interesting for designers of $\mathrm{HF}$ circuit, where the frequency dependence of components including conductive joints can be very significant for correct operation of HF equipment.

\section{REFERENCES}

[1] Agilent Impedance Measurement Handbook. A guide to measurement technology and techniques. $4^{\text {th }}$ edition, Agilent technologies

[2] MacDonald, J. R.: Impedance Spectroscopy: Old Problems and New Developments. Electrochimica Acta. Vol. 35. No. 10. Pp.1483 - 1492

[3] Hughes, CH.: Impedance Measurement. Syn-Aud-Con Newsletter. Vol. 35. No. 3. 2007. Pp. 7 - 12

[4] Dumbrava V., Svilajnis L.: The automated Complex Impedance Measurement System. Electronics and Electrical Engineering. No 4/76. 2007. Pp 59 - 62

\section{ACKNOWLEDGMENTS}

The work has been supported by a project "Diagnostics of Materials", number MSM6840770021 